\title{
TED, cela vous dit-il quelque chose?
}

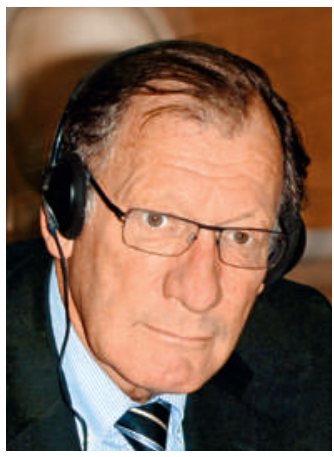

Jean Martin
A moi, ce terme ne me disait rien jusqu'il y a peu, quand un de nos enfants m'a fait visionner une présentation d'Amanda Palmer, musicienne américaine très originale. En février dernier, un événement TED était organisé à l'Université de Lausanne et j'ai voulu y participer. Bien intéressant.

TED est l'abréviation de Technology Entertainment Design, ce qui reste assez énigmatique. Initiative lancée dès 1984 mais qui s'est vraiment développée au $21^{\text {e }}$ siècle. Une TED Conférence (exposés successifs de 18 minutes maximum) est organisée annuellement en Californie et une TEDGlobal ailleurs, annuellement aussi, jusqu'ici surtout à Oxford et Edimbourg. Depuis 2009, il y a aussi un TEDMED traitant de technologie médicale. Le chapeau conceptuel: des idées qu'il vaut la peine de disséminer (ideas worth spreading).

Difficile de caractériser un TED. Peut-être des citations de la presse internationale de 2012 sont-elles utiles: «TED est une organisation mondiale spécialisée en conférences et en vidéos inspirationnelles mieux adaptée à l'éducation au $21^{\text {e }}$ siècle peut-être» (NY Times); «TED a cassé le moule de ce qu'on attend d'un exposé. Les orateurs n'expliquent pas, par exemple, pourquoi un produit est supérieur à un autre, ils cherchent plus à reconfigurer l'esprit des auditeurs» (The Economist); «TED réinvente l'accès à la connaissance et ressuscite l'échange d'idées par la parole» (Le Monde); «TED a grandi d'une petite niche pour devenir une global brand. Mais dans son essence cela reste une conférence où des histoires sont racontées sur une scène» (BBC News); «Il n'y pas beaucoup de réunions où un exposé de Bill Gates sur la prévention du paludisme est suivie par un discours sur la pornographie qui est en train de changer les relations entre les sexes. Bienvenue à TED.»

Autres avis: une place pour entrevoir le futur; rassemblant des CEO et des scientifiques, des artistes et d'autres qui défient toute catégorisation; qui sont là pour écouter des exposés ébouriffants (mindblowing); les vidéos TED ont réussi à convaincre la génération YouTube de s'asseoir et écouter; un carnaval intellectuel; l'ouverture radicale aux idées susceptibles de changer le monde.

Ces évènements sont organisés par des groupes privés de volontaires. Le TED lausannois récent avait pour titre «Perpetual (R)evolution» et a rempli le plus grand auditoire de l'UNIL. Des orateurs jeunes ayant lancé des entreprises ou projets originaux: un mili- tant qui promeut des «climate-friendly meals» dans les restaurants; une spécialiste étudiant au CHUV les horloges internes de notre cerveau; un scientifique démontrant de nouvelles approches de la réhabilitation des accidents vasculaires cérébraux; une femme qui rencontre le succès en Allemagne en communiquant à des sourds, par la langue des signes et le mouvement, les sensations suscitées par la musique (surprenant, non?); un artiste polyvalent qui s'intéresse de façons très originales au sens olfactif de nos contemporains; une Africaine soulignant qu'il faut d'abord changer son environnement local pour aller ensuite vers le global; un enseignant de l'éthique des affaires parlant de la prise de responsabilité corporative aussi bien qu'individuelle; un ancien enfant peureux qui nage maintenant au milieu des requins géants; une enseignante d'anthropologie dont la passion est la démocratie au quotidien et qui est certaine qu'on peut agir contre ce qui paraît inévitable.

Et puis des artistes. Notamment une Israélienne qui chante «Souvenons-nous que chaque poing fermé était une fois une main ouverte» et se fait l'avocate du vivre-ensemble et de la paix. Même si (autre chanson) «cela va devenir beaucoup pire encore avant que cela aille mieux».

A boire et à manger donc, mais de qualité... Etonnante diversité des préoccupations, des approches et des manières d'en parler. Echanges à la pause dans des ateliers sur des thèmes spécifiques. Et, au-delà des présentations physiques, la dissémination des messages par des vidéos librement accessibles sur le web (des milliers d'entre elles, de 130 pays).

Entre autres, TED est une illustration de transdisciplinarité, grand mot de notre temps. Interpellant tout en étant impeccable quant à la forme: l'allure, la diction, les textes des présentateurs sont très soignés, ont été répétés. Avec même des points qu'on n'attend pas: lors de l'inscription, il était indiqué que les participants étaient censés être en tenue de ville ou sport plutôt chic! On veut de l'original, du nouveau, du bousculant, de l'inspirational, avec une certaine rigueur.

Commentaire d'un de mes proches: «Des démonstrations que mon savoir n'existe pas sans ma vie»... Guère de doute que les évènements TED vont se multiplier . Quand vous en avez l'occasion, allez-y voir!

Jean Martin, membre de la rédaction 